\title{
Consistency conditions for the first-order formulation of Yang-Mills theory
}

\author{
D. G. C. McKeon $\odot^{*}$ \\ Department of Applied Mathematics, The University of Western Ontario, \\ London, Ontario N6A 5B7, Canada and Department of Mathematics and Computer Science, \\ Algoma University, Sault Ste. Marie, Ontario P6A 2G4, Canada \\ F. T. Brandt $\odot,^{\dagger}$ J. Frenkel, ${ }^{\dagger}$ and S. Martins-Filho $\circledast^{\S}$ \\ Instituto de Física, Universidade de São Paulo, São Paulo, SP 05508-090, Brazil
}

(Received 16 March 2020; accepted 1 April 2020; published 24 April 2020)

\begin{abstract}
We examine the self-consistency of the first-order formulation of the Yang-Mills theory. By comparing the generating functional $Z$ before and after integrating out the additional field $F_{\mu \nu}^{a}$, we derive a set of structural identities that must be satisfied by the Green's functions at all orders. These identities, which hold in any dimension, are distinct from the usual Ward identities and are necessary for the internal consistency of the first-order formalism. They relate the Green's functions involving the fields $F_{\mu \nu}^{a}$, to Green's functions in the second-order formulation which contain the gluon strength tensor $f_{\mu \nu}^{a}$. In particular, such identities may provide a simple physical interpretation of the additional field $F_{\mu \nu}^{a}$.
\end{abstract}

DOI: $10.1103 /$ PhysRevD.101.085013

\section{INTRODUCTION}

The first-order formulation of gauge theories has a simple form that involves only cubic interactions of the gauge fields, which are momentum-independent. This simplifies the computations of the quantum corrections in the standard second-order gauge theories, which involve momentum-dependent three-point as well as higher-point vertices. It is well known that the first-order formulation may be achieved by introducing, for example in the YangMills theory, an auxiliary field $F_{\mu \nu}^{a}$ [1-9]. The corresponding first-order Lagrangian density may be written as

$$
\tilde{\mathcal{L}}=\frac{1}{4} F_{\mu \nu}^{a} F^{\mu \nu a}-\frac{1}{2} f_{\mu \nu}^{a} F^{\mu \nu a}
$$

where $f_{\mu \nu}^{a}$ is the gluon field strength tensor

$$
f_{\mu \nu}^{a}=\partial_{\mu} A_{\nu}^{a}-\partial_{\nu} A_{\mu}^{a}+g f^{a b c} A_{\mu}^{b} A_{\nu}^{c} .
$$

Using the Euler-Lagrange equation in conjunction with the above Lagrangian, one can see that, at the classical level,

\footnotetext{
dgmckeo2@uwo.ca

†brandt@usp.br

*jfrenkel@if.usp.br

ssergiomartinsfilho@usp.br
}

Published by the American Physical Society under the terms of the Creative Commons Attribution 4.0 International license. Further distribution of this work must maintain attribution to the author(s) and the published article's title, journal citation, and DOI. Funded by SCOAP ${ }^{3}$.
$F_{\mu \nu}^{a}=f_{\mu \nu}^{a}$. From this it follows that $\tilde{\mathcal{L}}=-1 / 4 f_{\mu \nu}^{a} f^{a \mu \nu}$, which corresponds to the usual second-order Lagrangian.

At the quantum level, the renormalization of the firstorder formalism has been previously studied from various points of view [1-9]. In particular, the BRST renormalization of this formulation has been addressed in $[7,8]$. The BRST identities, which reflect the gauge invariance of the theory, are suitable for a recursive proof of the renormalizability to all orders in perturbation theory [10-12].

In the present work, we examine different kinds of identities, which are necessary for the consistency of the first-order formulation. To this end, we introduce a source $j_{\mu}^{a}$ for the gluon field $A_{\mu}^{a}$ and also a source $J_{\mu \nu}^{a}$ for the field $F_{\mu \nu}^{a}$, and consider the generating functional $Z[J, j]$ of Green's functions. We compare the functional dependence of $Z$ on the sources in the original first-order formalism with that found after making a shift

$$
F_{\mu \nu}^{a} \rightarrow F_{\mu \nu}^{a}+f_{\mu \nu}^{a}-2 J_{\mu \nu}^{a}
$$

which enables us to integrate out the auxiliary field $F_{\mu \nu}^{a}$. The equality of these functional forms leads to a set of structural identities among the Green's functions which must be satisfied to all orders, in any dimension. These show that in the first-order formalism, Green's functions containing only external gluon fields are the same as those which occur in the second-order formulation. Furthermore, these identities relate the Green's functions with some external $F_{\mu \nu}^{a}$ fields to certain Green's functions in the second-order formalism that contain the gluon strength 
tensor field $f_{\mu \nu}^{a}$. Such Green's functions involve composite fields, in which the external legs are "pinched" at the same spacetime point. As is well known, these lead to ultraviolet (short-distance) singularities [13-15]. In our case, such singularities play an important role. They are essential for the cancellation of ultraviolet divergences arising from loop diagrams, which is necessary for the implementation of the structural identities. Proceeding in this way, one obtains, in particular, the identity

$$
\begin{aligned}
& \left\langle 0\left|T F_{\mu \nu}^{a}(x) A_{\alpha_{1}}^{b_{1}}\left(x_{1}\right) \cdots A_{\alpha_{n}}^{b_{n}}\left(x_{n}\right)\right| 0\right\rangle \\
& \quad=\left\langle 0\left|T f_{\mu \nu}^{a}(x) A_{\alpha_{1}}^{b_{1}}\left(x_{1}\right) \cdots A_{\alpha_{n}}^{b_{n}}\left(x_{n}\right)\right| 0\right\rangle .
\end{aligned}
$$

This may be interpreted as the quantum-mechanical generalization of the relation $F_{\mu \nu}^{a}=f_{\mu \nu}^{a}$ obtained at the classical level. Namely, the Green's functions containing one $F_{\mu \nu}^{a}$ field and an arbitrary number of Yang-Mills fields are equal to the Green's functions involving a single gluon field strength tensor $f_{\mu \nu}^{a}$ and an arbitrary number of YangMills fields.

In Sec. II we analyze the Lagrangian and the generating functional of Green's functions in the first-order formulation. In Sec. III, we proceed to derive the result (1.4), which has been explicitly verified up to one-loop order. In Sec. IV, we study a basic structural identity satisfied by the Green's functions and examine the cancellations between the loop ultraviolet divergences and the ultraviolet singularities arising from tree graphs involving composite fields. We present a brief discussion of the results in Sec. V. Some details of the relevant calculations are given in the Appendices.

\section{THE LAGRANGIAN AND THE GENERATING FUNCTIONAL}

The complete Lagrangian density for the first-order formulation in covariant gauges is

$\mathcal{L}=\frac{1}{4} F_{\mu \nu}^{a} F^{\mu \nu a}-\frac{1}{2} f_{\mu \nu}^{a} F^{\mu \nu a}-\frac{1}{2 \xi}\left(\partial_{\mu} A^{\mu a}\right)^{2}+\left(\partial_{\mu} \bar{\eta}^{a}\right) D^{\mu a b} \eta^{b}$,

where $\xi$ is a gauge fixing parameter, $\bar{\eta}^{a}, \eta^{b}$ are ghost fields and $D^{\mu a b}$ is the covariant derivative

$$
D^{\mu a b}=\delta^{a b} \partial^{\mu}-g f^{a b c} A^{\mu c} .
$$

In addition, we will also introduce the external sources $J_{\mu \nu}^{a}$ and $j_{\mu}^{a}$ as follows

$$
\mathcal{L}_{\text {source }}=J_{\mu \nu}^{a} F^{\mu \nu a}+j_{\mu}^{a} A^{\mu a} .
$$

The normalized generating functional for Green's functions is given by

$Z[J, j]=\frac{\int \mathcal{D} \eta \mathcal{D} \bar{\eta} \mathcal{D} F \mathcal{D} A \exp i\left[S+\int d^{d} x\left(J_{\mu \nu}^{a} F^{\mu \nu a}+j_{\mu}^{a} A^{\mu a}\right)\right]}{\int \mathcal{D} \eta \mathcal{D} \bar{\eta} \mathcal{D} F \mathcal{D} A \exp i S}$,

where $S=\int d^{d} x \mathcal{L}$. This equation is in a form suitable for functional differentiation with respect to $J$ and $j$, and therefore for finding the Green's functions.

If we were to set $J_{\mu \nu}^{a}=0$ at the outset (so that we would consider Green's functions with only external fields $A_{\mu}^{a}$ ) and make the change of variable in the functional integral

$$
F_{\mu \nu}^{a} \rightarrow F_{\mu \nu}^{a}+f_{\mu \nu}^{a}
$$

then we find that

$$
Z[J=0, j]=Z_{2}[j]
$$

where $Z_{2}[j]$ is the generating functional for the secondorder theory, characterized by the Lagrangian density

$\mathcal{L}_{2}=-\frac{1}{4} f_{\mu \nu}^{a} f^{\mu \nu a}-\frac{1}{2 \xi}\left(\partial_{\mu} A^{\mu a}\right)^{2}+\left(\partial_{\mu} \bar{\eta}^{a}\right) D^{\mu a b} \eta^{b}$

together with the source term $j_{\mu}^{a} A^{\mu a}$. This establishes the important property that the Green's functions with only external gluon fields are the same in both approaches.

We now consider using $Z[J, j]$ with $J \neq 0$ and examine what changes occur in the first-order formalism when there are external fields $F_{\mu \nu}^{a}$. To this end we make the shift (1.3) in the numerator of (2.4) which leads, after integrating out the $F$ field, to the alternative form of the generating functional

$$
Z^{\prime}[J, j]=\frac{\int \mathcal{D} \eta \mathcal{D} \bar{\eta} \mathcal{D} A \exp i \int d^{d} x\left(\mathcal{L}_{2}+J_{\mu \nu}^{a} f^{\mu \nu a}-J_{\mu \nu}^{a} J^{\mu \nu a}+j_{\mu}^{a} A^{\mu a}\right)}{\int \mathcal{D} \eta \mathcal{D} \bar{\eta} \mathcal{D} A \exp i \int d^{d} x \mathcal{L}_{2}} .
$$

This equals to $Z_{2}[j]$ in Eq. (2.6) if we set $J_{\mu \nu}^{a}=0$. It is worth noticing here the unusual dependence of $Z^{\prime}[J, j]$ on $J_{\mu \nu}^{a}$.

Comparing the forms (2.4) and (2.8) of the generating functionals and differentiating these with respect to $J$ and $j$, leads to a set of structural identities among the Green's functions, which must be satisfied to all orders. In principle, the Green's functions obtained in this way should be evaluated by using the Feynman rules appropriate to the first-order formalism. However, since Green's functions 


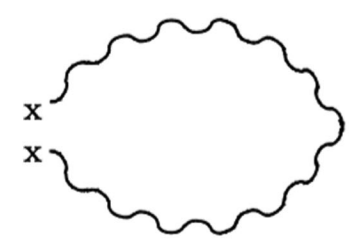

(a)

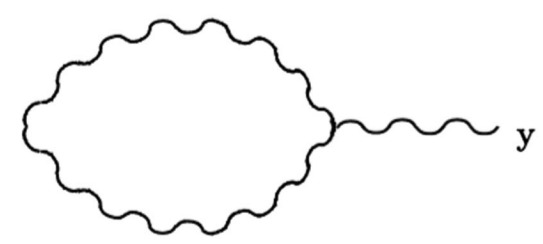

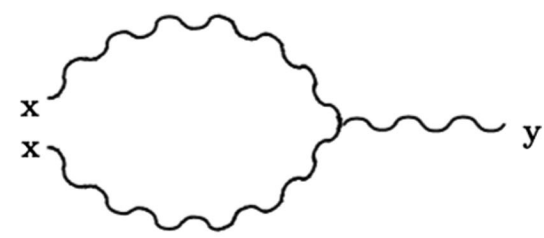

(b)

FIG. 1. Tadpole (a) and "pinched" contributions (b) from $\left\langle 0\left|T f^{a c d} A_{\mu}^{c}(x) A_{\nu}^{d}(x) A_{\alpha}^{b}(y)\right| 0\right\rangle$.

with only external gluon fields are the same as those in the second-order formulation, the Green's functions obtained via Eq. (2.8) are equal to the corresponding ones obtained by using this formulation. Therefore, we see that such structural identities may relate the Green's functions involving some $F$ fields to certain Green's functions in the second-order formulation that contain gluon strength tensor fields $f_{\mu \nu}^{a}$. These identities hold in any dimensions, both for the finite as well as for the ultraviolet divergent parts of the Green's functions.

\section{DERIVATION OF RELATION (1.4)}

Taking the functional differentiation of Eqs. (2.4) and (2.8) with respect to $J$ and $j$, and equating the results we obtain, by setting $J=j=0$, the equation

$$
\left\langle 0\left|T F_{\mu \nu}^{a}(x) A_{\alpha}^{b}(y)\right| 0\right\rangle=\left\langle 0\left|T f_{\mu \nu}^{a}(x) A_{\alpha}^{b}(y)\right| 0\right\rangle .
$$

Using the Feynman rules given in Appendix A one can verify that this equation, which relates the propagators $F A$ and $f A$, is satisfied in the tree approximation. To one-loop order, the divergent part of the left-hand side in momentum space is [see Eq. (B39)]

$D_{F A \mu \nu, \alpha}^{(1) a b}=\frac{C_{\mathrm{YM}} g^{2}}{16 \pi^{2} \epsilon} \delta^{a b} \frac{11-9 \xi}{12} \frac{1}{k^{2}}\left(k_{\mu} \eta_{\nu \alpha}-k_{\nu} \eta_{\mu \alpha}\right)$,

where we have used dimensional regularization in $d=$ $4-2 \epsilon$ dimensions. Our conventions are such that the configuration space derivative $\partial_{\mu}$ becomes in momentum space $+i k$ where the momentum $k$ is flowing into the vertex with which it is associated.

We must now calculate the divergent part of the propagator on the right-hand side of Eq. (3.1). One contribution to this comes from the $\partial_{\mu} A_{\nu}^{a}-\partial_{\nu} A_{\mu}^{a}$ part of $f_{\mu \nu}^{a}$. With the help of the Eq. (B36), this part yields in momentum space

$$
\frac{C_{\mathrm{YM}} g^{2}}{16 \pi^{2} \epsilon} \delta^{a b} \frac{13-3 \xi}{6} \frac{1}{k^{2}}\left(k_{\mu} \eta_{\nu \alpha}-k_{\nu} \eta_{\mu \alpha}\right)
$$

The other contribution comes from the composite field $g f^{a b c} A_{\mu}^{b}(x) A_{\nu}^{c}(x)$ which occurs in $f_{\mu \nu}^{a}(x)$. Using Wick's theorem, one can verify that to order $g^{2}$, such a term arises from the Feynman diagrams shown in Fig. 1. The first graph contains a tadpole, Fig. 1(a), which vanishes by using dimensional regularization. The second diagram corresponds to a three-point tree Green's function which has however two coordinates "pinched" at the same spacetime point $x$. As noted earlier, such a composite field leads to an ultraviolet (short-distance) singularity (see also Appendix B). Using the well known expression for the three-point gluon vertex, it is straightforward to evaluate this contribution in momentum space, which turns out to be [see Eq. (B42)]

$$
-\frac{C_{\mathrm{YM}} g^{2}}{16 \pi^{2} \epsilon} \delta^{a b} \frac{\xi+5}{4} \frac{1}{k^{2}}\left(k_{\mu} \eta_{\nu \alpha}-k_{\nu} \eta_{\mu \alpha}\right) .
$$

Adding the contributions given in Eqs. (3.3) and (3.4), we obtain a result which agrees with that given in Eq. (3.2). Thus, we have explicitly verified, to one-loop order, the validity of the identity (3.1) for the UV divergent parts (in Appendix B we show that this is valid for the full expression in $d$ dimensions). It is straightforward to generalize Eq. (3.1) so as to include an arbitrary number of gluon fields, namely

$$
\begin{aligned}
& \left\langle 0\left|T F_{\mu \nu}^{a}(x) A_{\alpha_{1}}^{b_{1}}\left(x_{1}\right) \cdots A_{\alpha_{n}}^{b_{n}}\left(x_{n}\right)\right| 0\right\rangle \\
& \quad=\left\langle 0\left|T f_{\mu \nu}^{a}(x) A_{\alpha_{1}}^{b_{1}}\left(x_{1}\right) \cdots A_{\alpha_{n}}^{b_{n}}\left(x_{n}\right)\right| 0\right\rangle .
\end{aligned}
$$

As we have mentioned, this relation may be interpreted as being a quantum-mechanical extension of the relation $F_{\mu \nu}^{a}=$ $f_{\mu \nu}^{a}$ which holds at the classical level.

\section{A BASIC STRUCTURAL IDENTITY}

Applying $\delta^{2} / \delta J_{\mu \nu}^{a}(x) \delta J_{\alpha \beta}^{b}(y)$ to Eqs. (2.4) and (2.8) and equating the results, leads to

$$
\begin{aligned}
& \left\langle 0\left|T F_{\mu \nu}^{a}(x) F_{\alpha \beta}^{b}(y)\right| 0\right\rangle \\
& \quad=2 i \delta^{a b} I_{\mu \nu, \alpha \beta} \delta^{4}(x-y)+\left\langle 0\left|T f_{\mu \nu}^{a}(x) f_{\alpha \beta}^{b}(y)\right| 0\right\rangle,
\end{aligned}
$$

where $I_{\mu \nu, \alpha \beta}$ is given by Eq. (A2). As we have explained following Eq. (2.8), the above equation relates the propagators $\left\langle 0\left|T F_{\mu \nu}^{a}(x) F_{\alpha \beta}^{b}(y)\right| 0\right\rangle$ calculated in the first-order formalism to the Green's functions $\left\langle 0\left|T f_{\mu \nu}^{a}(x) f_{\alpha \beta}^{b}(y)\right| 0\right\rangle$ computed in the second-order formalism. We now will examine the perturbative expansion of each side of Eq. (4.1). It is easy to verify that this equation is satisfied at the tree level. To one-loop order, using the Feynman rules given in Appendix A, one can show [see Eq. (B24)] that the graphs that contribute to the left side (see Fig. 4) yield in momentum space the pole term 


$$
\begin{aligned}
D_{F F \mu \nu, \alpha \beta}^{(1) a b}= & i \frac{C_{\mathrm{YM}} g^{2}}{16 \pi^{2} \epsilon} \delta^{a b}\left\{-(\xi+1) I_{\mu \nu, \alpha \beta}\right. \\
& \left.+\left(\frac{2}{3}+2 \xi\right) \frac{1}{k^{2}} L_{\mu \nu, \alpha \beta}(k)\right\}+\cdots,
\end{aligned}
$$

where $L_{\mu \nu, \alpha \beta}(k)$ is given by Eq. (A3) and we use dimensional regularization in $4-2 \epsilon$ dimensions.

The computation of the right-hand side of Eq. (4.1) is somewhat more involved at order $g^{2}$, where we encounter contributions from three sources. The first one, which corresponds to $\left\langle 0\left|T\left(\partial_{\mu} A_{\nu}^{a}-\partial_{\nu} A_{\mu}^{a}\right)(x)\left(\partial_{\alpha} A_{\beta}^{b}-\partial_{\beta} A_{\alpha}^{b}\right)(y)\right| 0\right\rangle$ comes from one-loop graphs shown in Fig. 2. This yields in momentum space the pole term [see Eq. (B32)]

$$
i \frac{C_{\mathrm{YM}} g^{2}}{16 \pi^{2} \epsilon} \delta^{a b} \frac{3 \xi-13}{3} \frac{1}{k^{2}} L_{\mu \nu, \alpha \beta}(k) .
$$

The other contributions arise from the composite fields which occur in the Green's functions $g f^{b c d}\langle 0| T\left(\partial_{\mu} A_{\nu}^{a}-\right.$ $\left.\partial_{\nu} A_{\mu}^{a}\right) A_{\alpha}^{c} A_{\beta}^{d}|0\rangle, \quad g f^{a c d}\left\langle 0\left|T A_{\mu}^{c} A_{\nu}^{d}\left(\partial_{\alpha} A_{\beta}^{b}-\partial_{\beta} A_{\alpha}^{b}\right)\right| 0\right\rangle \quad$ and $g^{2} f^{a c d} f^{b c^{\prime} d^{\prime}}\left\langle 0\left|T A_{\mu}^{c} A_{\nu}^{d} A_{\alpha}^{c^{\prime}} A_{\beta}^{d^{\prime}}\right| 0\right\rangle$. Since the tadpole graphs vanish when using dimensional regularization, the only Feynman diagrams which contribute to these Green's functions are shown in Figs. 5(a)-5(c) respectively. These yield, in momentum space, the following pole terms [see Eqs. (B44) and (B46)]

$$
i \frac{g^{2} C_{\mathrm{YM}}}{16 \pi^{2} \epsilon} \delta^{a b}(\xi+5) \frac{1}{k^{2}} L_{\mu \nu, \alpha \beta}(k)
$$

and

$$
-i \frac{g^{2} C_{\mathrm{YM}}}{16 \pi^{2} \epsilon} \delta^{a b}(\xi+1) I_{\mu \nu, \alpha \beta} .
$$

There is an aspect of the contributions from Fig. 5 that is worth pointing out. The divergent terms given respectively by Eqs. (4.4) and (4.5), come from "pinching" at the same spacetime point the legs of what would otherwise be a tree diagram. Explicit calculation of such diagrams once the external legs are "pinched," gives rise to short distance (ultraviolet) singularities as $\epsilon \rightarrow 0$ when using dimensional regularization. Adding the contributions coming from Eqs. (4.3), (4.4) and (4.5) leads to the result (4.2), thereby verifying the pole part of the identity (4.1) to order $g^{2}$ (in Appendix B we show that this is valid for the full expression in $d$ dimensions).

\section{DISCUSSION}

We have studied certain consistency conditions for the first-order formulation of the Yang-Mills theory. To this end, we examined the forms of the generating functionals of Green's functions $Z(J, j)$, before and after integrating out the additional field $F_{\mu \nu}^{a}$. Differentiations of these forms with respect to $J_{\mu \nu}^{a}$ and $j_{\mu}^{a}$ yield a set of structural identities which are complementary but distinct from the usual Ward identities. Such identities lead to connections between the Green's functions involving the field $F_{\mu \nu}^{a}$ and the Green's functions in the second-order formulation that contain the gluon strength tensor $f_{\mu \nu}^{a}$. An interesting outcome of these relations is a quantum-mechanical extension of the classical result $F_{\mu \nu}^{a}=f_{\mu \nu}^{a}$, which provides a simple interpretation of the field $F_{\mu \nu}^{a}$.

The structural identities hold for the complete Green's functions, in any dimensions and to all orders. We have explicitly verified such identities to one loop-order, for the ultraviolet divergent parts. These require subtle cancellations between the ultraviolet divergences coming from loop graphs and the short-distance singularities induced by the composite fields present in the gluon strength tensor $f_{\mu \nu}^{a}$. These results provide a simpler computation of the expectation values of time-ordered products of operators containing the composite gluon strength tensor $f_{\mu \nu}^{a}$, in terms of those involving the local field $F_{\mu \nu}^{a}$.

It is known that the renormalizability of the first-order formulation requires, as well as a scaling of the $F_{\mu \nu}^{a}$ field, also a mixing with the gluon strength tensor field $f_{\mu \nu}^{a}$

$$
F_{\mu \nu}^{a} \rightarrow Z_{F}^{1 / 2} F_{\mu \nu}^{a}+Z_{F f} f_{\mu \nu}^{a}
$$

where $Z_{F f}$ is a counterterm which is equal to $(1-3 \xi) g^{2} C_{\mathrm{YM}} / 192 \pi^{2} \epsilon$, at one-loop order [7]. Hence, one may also expect a scaling and mixing of sources of the form

$$
j_{\mu}^{a} \rightarrow Z_{j}^{1 / 2} j_{\mu}^{a}+z D^{\nu a b} J_{\mu \nu}^{b}
$$

which is admissible on dimensional, Lorentz and chargeconjugation symmetry grounds. Yet, our explicit one-loop calculations show that $z=0$. This result may be understood by noting that the last term in Eq. (5.2) could induce corrections which would violate the Eq. (4.1). Thus we infer that, to all orders, the structural identities forbid a mixing between the sources $j_{\mu}^{a}$ and $J_{\mu \nu}^{a}$.

Finally, we remark that the first-order formalism is also useful in quantum gravity, where it allows us to replace an infinite number of complicated multiple graviton couplings by a finite number of simple cubic vertices $[5,6]$. In this theory, one would similarly get corresponding structural identities, which ensure the internal consistency of such a formulation. This is an interesting issue which deserves further study.

\section{ACKNOWLEDGMENTS}

F. T. B. and J.F. thank CNPq (Brazil) for financial support. S. M.-F. thanks CAPES (Brazil) for financial support. D. G. C. M. thanks Roger Macleod for an enlightening discussion. This work comes as an aftermath of an original project developed with the support of FAPESP (Brazil), Grant No. 2018/01073-5. 


\section{APPENDIX A: FEYNMAN RULES}

The following Feynman rules for the first-order YangMills theory can be readily obtained from the Lagrangian density in Eq. (2.1) (for details, see Ref. [5])

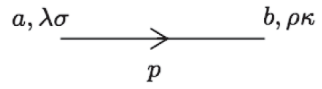

$2 i\left(I_{\lambda \sigma, \rho \kappa}-\frac{1}{p^{2}} L_{\lambda \sigma, \rho \kappa}(p)\right) \delta^{a b}$,

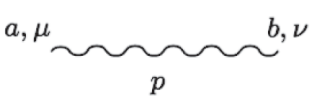

$-\frac{i}{p^{2}}\left(\eta_{\mu \nu}-\frac{1-\xi}{p^{2}} p_{\mu} p_{\nu}\right) \delta^{a b}$

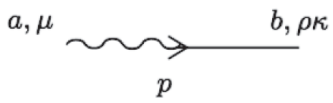

$-\frac{1}{p^{2}}\left(p_{\rho} \eta_{\kappa \mu}-p_{\kappa} \eta_{\rho \mu}\right) \delta^{a b}$

(A1c)

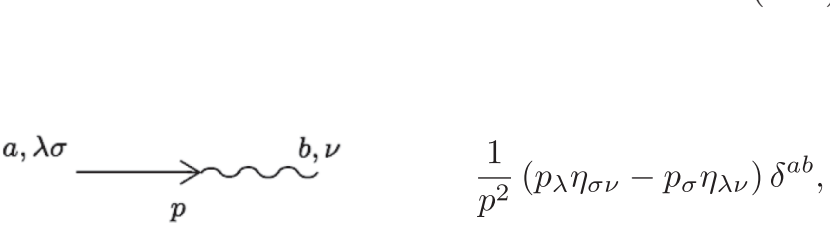

(A1d)

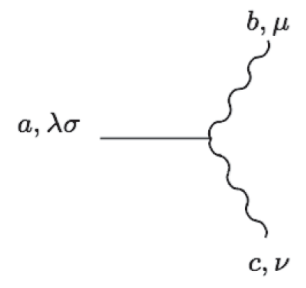

$$
-i \frac{g}{2} f^{a b c}\left(\eta_{\lambda \mu} \eta_{\sigma \nu}-\eta_{\sigma \mu} \eta_{\lambda \nu}\right)
$$

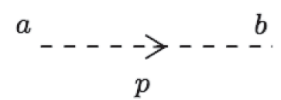

$$
\frac{i}{p^{2}} \delta^{a b}
$$

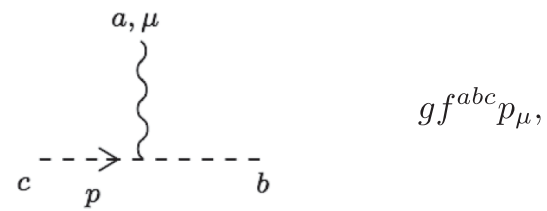

$(\mathrm{A} 1 \mathrm{~g})$ where the quanta of the $A_{\mu}^{a}$ and $F_{\mu \nu}^{a}$ fields are represented respectively by the wavy and the continuous lines. Here, the tensors $I_{\mu \nu, \alpha \beta}$ and $L_{\mu \nu, \alpha \beta}(p)$ are given in momentum space by

$$
I_{\mu \nu, \alpha \beta}=\frac{1}{2}\left(\eta_{\mu \alpha} \eta_{\nu \beta}-\eta_{\nu \alpha} \eta_{\mu \beta}\right)
$$

and

$$
L_{\mu \nu, \alpha \beta}(p)=\frac{1}{2}\left(p_{\mu} p_{\alpha} \eta_{\nu \beta}+p_{\nu} p_{\beta} \eta_{\mu \alpha}-p_{\nu} p_{\alpha} \eta_{\mu \beta}-p_{\mu} p_{\beta} \eta_{\nu \alpha}\right) .
$$

It is also convenient to denote the free propagators in Eqs. (A1a), (A1b), (A1c) and (A1d) respectively as $D_{F F \mu \nu, \alpha \beta}^{(0) a b}(p), D_{A A \mu \nu}^{(0) a b}(p), D_{A F \mu, \alpha \beta}^{(0) a b}(p)$ and $D_{F A \alpha \beta, \mu}^{(0) a b}(p)$. Note that the tensors (A2) and (A3) satisfy

$$
p^{\rho} I_{\mu \nu, \alpha \rho}=p^{\rho} \frac{1}{p^{2}} L_{\mu \nu, \alpha \rho}(p)=\frac{1}{2}\left(\eta_{\mu \alpha} p_{\nu}-\eta_{\nu \alpha} p_{\mu}\right)
$$

which imply that the $F$-propagator in (A1) satisfies the transversality condition

$$
p^{\mu} D_{F F \mu \nu, \alpha \beta}^{(0) a b}(p)=0 .
$$

Also, the identities

$I_{\mu \nu, \lambda \rho} \frac{1}{p^{2}} L_{\alpha \beta}^{\lambda \rho}(p)=\frac{1}{p^{2}} L_{\mu \nu, \lambda \rho}(p) \frac{1}{p^{2}} L_{\alpha \beta}^{\lambda \rho}(p)=\frac{1}{p^{2}} L_{\mu \nu, \alpha \beta}(p)$

$\left(L_{\mu \nu, \alpha \beta}(p) / p^{2}\right.$ is idempotent) imply that the $F$-propagator satisfies the relation

$$
L_{\mu \nu, \lambda \rho}(p) D_{F F}^{(0)} \underset{\alpha \beta}{\lambda \rho}(p)=0 .
$$

For completeness, let us also display the well-known Feynman rules obtained from the second-order formalism Lagrangian given by Eq. (2.7). The propagators for the $A_{\mu}^{a}$ and the ghost fields, as well as the ghost vertex, are the same as in Eqs. (A1b), (A1f) and (A1g). But now, instead of the single momentum independent vertex, given by (A1e), as well as the mixed propagator in Eq. (A1c), we have the following cubic and quartic vertices 


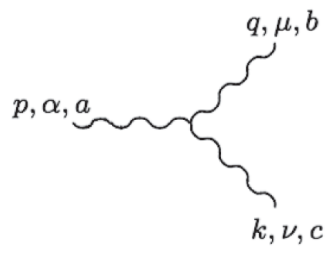

$$
g f^{a b c}\left[\left(p_{\nu}-q_{\nu}\right) \eta_{\mu \alpha}+\left(q_{\alpha}-k_{\alpha}\right) \eta_{\mu \nu}+\left(k_{\mu}-p_{\mu}\right) \eta_{\alpha \nu}\right],
$$

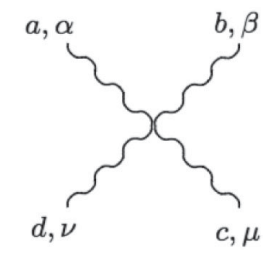

$c, \mu$

$$
\begin{array}{r}
-i g^{2}\left[f^{a b e} f^{c d e}\left(\eta_{\alpha \mu} \eta_{\beta \nu}-\eta_{\beta \mu} \eta_{\alpha \nu}\right)+\right. \\
f^{a c e} f^{d b e}\left(\eta_{\alpha \nu} \eta_{\mu \beta}-\eta_{\mu \nu} \eta_{\alpha \beta}\right)+ \\
\left.f^{\text {ade }} f^{b c e}\left(\eta_{\alpha \beta} \eta_{\nu \mu}-\eta_{\nu \beta} \eta_{\alpha \mu}\right)\right]
\end{array}
$$

with all momenta flowing inwards.

The identities (3.1) and (4.1) can be verified at the lowest order, in the momentum space, using the free propagators introduced in the Feynman rules above. The tree level momentum space version of Eq. (3.1)

$$
D_{F A \lambda \sigma, \nu}^{(0) a b}=i p_{\lambda} D_{A A \sigma \nu}^{(0) a b}-i p_{\sigma} D_{A A \lambda \nu}^{(0) a b}
$$

is verified using Eqs. (A1d) and (A1b) (note that the momentum space expression of the bilinears like $(\partial A(x) \cdots A(x))$ is $i p \tilde{A}(p) \cdots \tilde{A}(-p))$.

Similarly, the momentum space form of Eq. (4.1) can be written as

$$
\begin{aligned}
D_{F F \mu \nu, \alpha \beta}^{(0) a b}= & 2 i \delta^{a b} I_{\mu \nu, \alpha \beta}+p_{\mu} p_{\alpha} D_{A A \nu \beta}^{(0) a b}-p_{\mu} p_{\beta} D_{A A \nu \alpha}^{(0) a b} \\
& -p_{\nu} p_{\alpha} D_{A A \mu \beta}^{(0) a b}+p_{\nu} p_{\beta} D_{A A \mu \alpha}^{(0) a b},
\end{aligned}
$$

which can be readily verified using the Eqs. (A1a) and (A1b).

\section{APPENDIX B: ONE-LOOP RESULTS}

\section{Self-energies}

\section{a. The general method and the $A_{\mu}^{a}$ self-energy in the second-order formalism}

Let us first consider all the possible self-energy diagrams that can be computed using the Feynman rules presented in Appendix A. As is well known, these basic 1PI diagrams are the basic building blocks that contribute to the identities like the ones given by Eqs. (3.1) and (4.1).

The diagrams which contribute to the well known result for $A$ field self-energy, in the second-order formalism, are shown in Fig. 2 (diagrams in Figs. 2(a), 2(c) and 4(c) have a combinatorial factor $1 / 2$; there is a minus sign for the ghost loop diagrams). Figure 3 show the contributions to the $A$ field self-energy in the first-order formalism. Our basic approach for the computation of all the self-energies will be based on tensor decompositions. In the case of Figs. 2 and 3 , all the diagrams will have, after the loop momentum integration, the following covariant tensor structure

$$
\begin{aligned}
\Pi_{\mu \nu}^{I a b}(k) & =C_{\mathrm{YM}} \delta^{a b}\left(C_{1}^{I} \eta_{\mu \nu}+C_{2}^{I} \frac{k_{\mu} k_{\nu}}{k^{2}}\right) ; \\
I & =(2 \mathrm{a}),(2 \mathrm{~b}),(2 \mathrm{c})
\end{aligned}
$$

(we are using $f^{a m n} f^{b m n}=C_{\mathrm{YM}} \delta^{a b}$ ).

The coefficients $C_{i}^{I}$ can be obtained solving the following system of two algebraic equations

$$
\left\{\begin{array}{l}
\eta^{\mu \nu} \Pi^{I}{ }_{\mu \nu}(k)=d C_{1}^{I}+C_{2}^{I} \\
k^{\mu} k^{\nu} \Pi^{I}{ }_{\mu \nu}(k)=k^{2} C_{1}^{I}+k^{2} C_{2}^{I}
\end{array} ; \quad I=(2 \mathrm{a}),(2 \mathrm{~b}) \text { and }(2 \mathrm{c}),\right.
$$

where we have introduced $\Pi_{\mu \nu}^{I}(k)$ (without the color indices) such that $\Pi_{\mu \nu}^{I a b}(k)=C_{\mathrm{YM}} \delta^{a b} \Pi_{\mu \nu}^{I}(k)$.

Using the Feynman rules given in Appendix A, the scalar integrals on the left-hand side of the Eqs. (B2) will have the following form

$$
\int \frac{d^{d} p}{(2 \pi)^{d}} s^{I}(p, q, k)
$$

where $q=p+k ; p$ is the loop momentum, $k$ is the external momentum and $s^{I}(p, q, k)$ are scalar functions of $p \cdot k, q \cdot k$, $p \cdot q, p^{2}, q^{2}$ and $k^{2}$. Upon using the following relations

$$
\begin{aligned}
& p \cdot k=\left(q^{2}-p^{2}-k^{2}\right) / 2, \\
& q \cdot k=\left(q^{2}+k^{2}-p^{2}\right) / 2, \\
& p \cdot q=\left(p^{2}+q^{2}-k^{2}\right) / 2,
\end{aligned}
$$




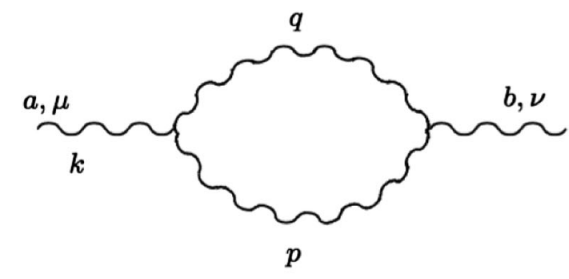

(a)

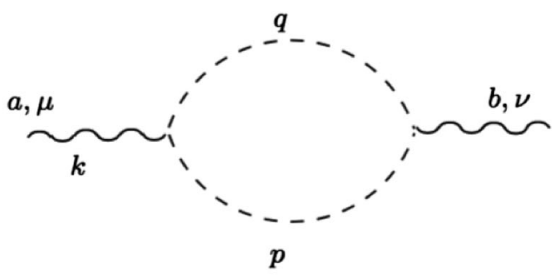

(b)

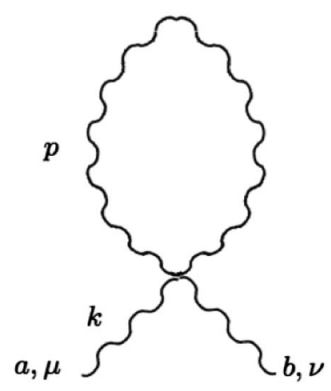

(c)

FIG. 2. One-loop contributions to the gauge field self-energy in the second-order formalism $(q=p+k$ and $k$ in an inward external momentum). There is a combinatorial $1 / 2$ factor associated with diagrams (a) and (c) and a minus sign associated with the ghost-loop diagram in (b). The relevant Feynman rules are given in Eqs. (A1b), (A1f), (A1g), (A8a) and (A8b).

the scalars $s^{I}(p, q, k)$ can be reduced to combinations of powers of $p^{2}$ and $q^{2}$. As a result, the integrals in Eq. (B3) can all be expressed in terms of combinations of the following simple integrals

$$
\begin{aligned}
I^{l m} \equiv & \int \frac{d^{d} p}{(2 \pi)^{d}} \frac{1}{\left(p^{2}\right)^{l}\left(q^{2}\right)^{m}} \\
= & i^{d+1} \frac{\left(k^{2}\right)^{d / 2-l-m}}{(4 \pi)^{d / 2}} \frac{\Gamma(l+m-d / 2)}{\Gamma(l) \Gamma(m)} \\
& \times \frac{\Gamma(d / 2-l) \Gamma(d / 2-m)}{\Gamma(d-l-m)},
\end{aligned}
$$

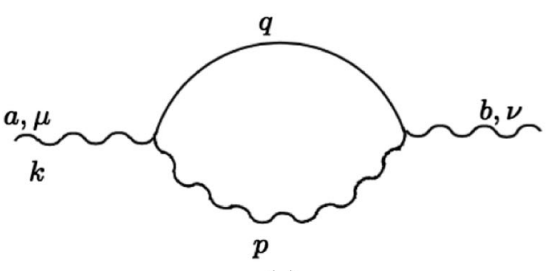

(a) where powers $l>1$ and $m>1$ may only arise from the terms proportional to $1-\xi$ in the gluon propagator [see Eq. (A1b)]. The only nonvanishing (i.e., nontadpole) integrals are

$$
\begin{gathered}
I^{11}=i^{d+1} \frac{\left(k^{2}\right)^{d / 2-2}}{2^{d} \pi^{d / 2}} \frac{\Gamma\left(2-\frac{d}{2}\right) \Gamma\left(\frac{d}{2}-1\right)^{2}}{\Gamma(d-2)} \\
I^{12}=I^{21}=\frac{(3-d)}{k^{2}} I^{11} \\
I^{22}=\frac{(3-d)(6-d)}{k^{4}} I^{11} .
\end{gathered}
$$

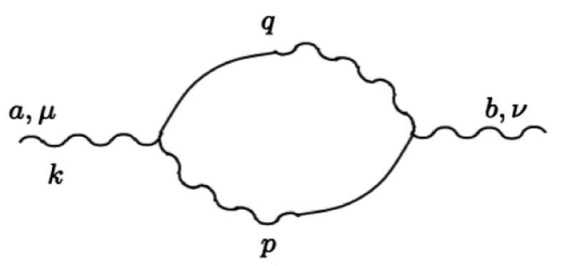

(b)

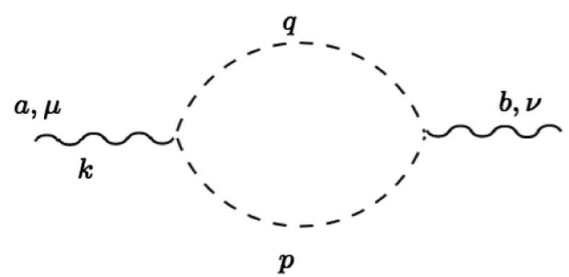

(c)

FIG. 3. One-loop contributions to the gauge field self-energy in the first-order formalism $(q=p+k$ and $k$ in an inward external momentum). The relevant Feynman rules are given in Eqs. (A1). 
In $d=4-2 \epsilon$ dimensions, $1 / \epsilon$ ultraviolet pole part of the basic integral $I^{11}$ is given by

$$
I^{11}=\frac{i}{16 \pi^{2} \epsilon} .
$$

Implementing the above-described procedure as a straightforward computer algebra code, we readily obtain the following results

$$
\left\{\begin{array}{l}
C_{1}^{(2 a)}=-\frac{k^{2}\left(d^{2}(\xi-1)(\xi+7)+d(19-\xi(5 \xi+26))+4 \xi(\xi+5)-14\right)}{8(d-1)} g^{2} I^{11}, \\
C_{2}^{(2 a)}=-C_{1}^{(2 a)}-\frac{k^{2}}{4} g^{2} I^{11}
\end{array},\right.
$$

$$
\begin{gathered}
\left\{\begin{array}{c}
C_{1}^{(2 b)}=\frac{k^{2}}{4(d-1)} g^{2} I^{11} \\
C_{2}^{(2 b)}=\frac{k^{2}}{4} g^{2} I^{11}-C_{1}^{(2 b)}
\end{array},\right. \\
C_{1}^{(2 c)}=C_{2}^{(2 c)}=0,
\end{gathered}
$$

where we have used the formulas in Eqs. (B6).

Equation (B8c) follows from the tadpole nature of the diagram in Fig. 2(c) which vanishes when one uses dimensional regularization. Notice that Eqs. (B8a), (B8b) and (B8c) imply that $C_{2}^{(2 a)}+C_{2}^{(2 b)}+C_{2}^{(2 c)}=-\left(C_{1}^{(2 a)}+\right.$ $C_{1}^{(2 b)}+C_{1}^{(2 c)}$, so that the self-energy will be transverse. The sum $C_{1}^{(2 a)}+C_{1}^{(2 b)}+C_{1}^{(2 c)}$ gives the following result

$$
\frac{d^{2}(1-\xi)(\xi+7)+d(\xi(5 \xi+26)-19)-4 \xi(\xi+5)+16}{8(d-1)} g^{2} I^{11} k^{2}
$$

Therefore, the final result for the $A_{\mu}^{a}$ field self-energy in the second-order formalism is given by

$$
\begin{aligned}
\Pi_{A A}^{(2 n d) a b}(k) & =\Pi_{\mu \nu}^{(2 a) a b}(k)+\Pi_{\mu \nu}^{(2 b) a b}(k)+\Pi_{\mu \nu}^{(2 c) a b}(k) \\
& =\frac{d^{2}(1-\xi)(\xi+7)+d(\xi(5 \xi+26)-19)-4 \xi(\xi+5)+16}{8(d-1)} g^{2} I^{11} C_{\mathrm{YM}} \delta^{a b}\left(k^{2} \eta_{\mu \nu}-k_{\mu} k_{\nu}\right)
\end{aligned}
$$

which is in agreement with the well-known result in $d$ dimensions (see Eq. (A.12) of [14] and the comment on the missing factor of $i$ on page 81). Using (B7) we obtain the following UV pole part $\left(g^{2} I^{11} \approx i g^{2} /\left(16 \pi^{2} \epsilon\right)\right)$

$$
\Pi_{A A}^{(2 n d) a b}{ }_{\mu \nu}(k)=i \frac{C_{\mathrm{YM}} g^{2}}{16 \pi^{2} \epsilon} \frac{13-3 \xi}{6} \delta^{a b}\left(k^{2} \eta_{\mu \nu}-k_{\mu} k_{\nu}\right)+\cdots,
$$

which is in agreement with the well-known result (see Eqs. (A.19) and (A.21) of [14]).

\section{b. $A_{\mu}^{a}$ self-energy in the first-order formalism}

Let us now consider the $A_{\mu}^{a}$ self-energy in the first-order formalism. The one-loop diagrams are shown in Fig. 3. Using the Feynman rules given in the Appendix $\mathrm{A}$ and considering that we have the same covariant structure as in Eq. (B1), Eqs. (B2) can be solved, with $I=(3 \mathrm{a}),(3 \mathrm{~b})$ and (3c), yielding following results

$$
\left\{\begin{array}{l}
C_{1}^{(3 a)}=\frac{k^{2}(d-2)}{4(d-1)}[d(1-\xi)+\xi-2] g^{2} I^{11} \\
C_{2}^{(3 a)}=\frac{k^{2}}{4}(d-2) g^{2} I^{11}-C_{1}^{(3 a)}
\end{array},\right.
$$

$$
\begin{aligned}
& \left\{\begin{array}{l}
C_{1}^{(3 b)}=-\frac{k^{2}}{4} g^{2} I^{11} \\
C_{2}^{(3 b)}=-\frac{k^{2}}{4}(d-1) g^{2} I^{11}-C_{1}^{(3 b)}
\end{array},\right. \\
& \left\{\begin{array}{l}
C_{1}^{(3 c)}=\frac{k^{2}}{4(d-1)} g^{2} I^{11} \\
C_{2}^{(3 c)}=\frac{k^{2}}{4} g^{2} I^{11}-C_{1}^{(3 c)}
\end{array}\right.
\end{aligned}
$$

where we have used Eqs. (B6).

As we can see the transversality condition is also satisfied in the first-order formalism. Indeed, Eqs. (B12a), (B12b) and (B12c), imply that $C_{2}^{(3 a)}+C_{2}^{(3 b)}+C_{2}^{(3 c)}=-\left(C_{1}^{(3 a)}+\right.$ $\left.C_{1}^{(3 b)}+C_{1}^{(3 c)}\right)$. From the sum $C_{1}^{(3 a)}+C_{1}^{(3 b)}+C_{1}^{(3 c)}$ we obtain the following result for the $A_{\mu}^{a}$ self-energy in the first-order formalism

$$
\begin{aligned}
& \Pi_{A A \mu \nu}^{(1 s t) a b}(k)=\Pi_{A A \mu \nu}^{(3 a) a b}(k)+\Pi_{A A \mu \nu}^{(3 b) a b}(k)+\Pi_{A A \mu \nu}^{(3 c) a b}(k) \\
& \quad=\frac{(d-2)[d(1-\xi)+\xi-3]}{4(d-1)} g^{2} I^{11} C_{\mathrm{YM}} \delta^{a b}\left(k^{2} \eta_{\mu \nu}-k_{\mu} k_{\nu}\right)
\end{aligned}
$$

which is different from the result in the second-order formalism, given by Eq. (B10). Using (B7) we obtain the following result for the UV pole 


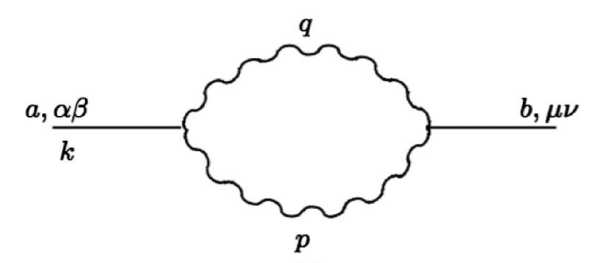

(a)
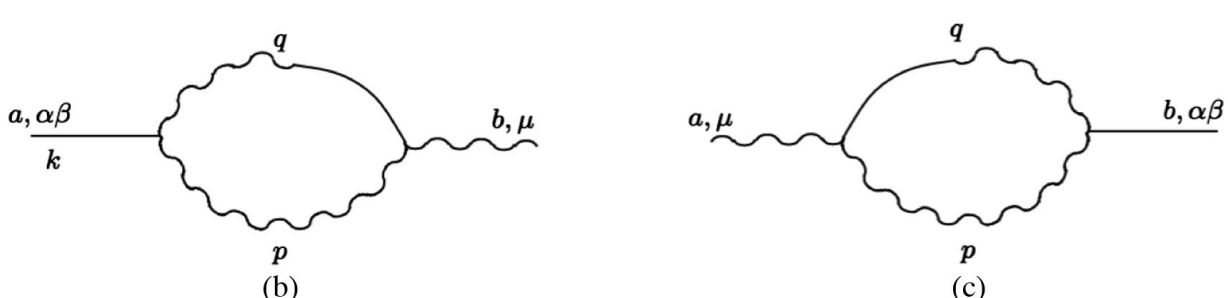

(c)

FIG. 4. One-loop contributions to the self-energies with external $F$ fields in the first-order formalism $(q=p+k$ and $k$ in an inward external momentum). The relevant Feynman rules are given in Eqs. (A1).

$\Pi_{A A \mu \nu}^{(1 s t) a b}(k)=i \frac{C_{\mathrm{YM}} g^{2}}{16 \pi^{2} \epsilon} \frac{1-3 \xi}{6} \delta^{a b}\left(k^{2} \eta_{\mu \nu}-k_{\mu} k_{\nu}\right)+\cdots$

Of course there is no contradiction with the general conclusions of Sec. II, which asserts that the two formalisms should have the same Green's functions containing only external $A_{\mu}^{a}$ fields; the 1PI functions are not necessarily the same in both formalisms. On the other hand, as we will see bellow from Eq. (B26), the propagator for the $A_{\mu}^{a}$ field is the same in both formalisms.

\section{c. $F_{\mu \nu}^{a}$ self-energy}

A complete tensor basis for the diagram in Fig. 4(a) can be formed using the two tensors introduced in Eqs. (A2) and (A3), since these are the most general tensors with four indices and having the required symmetry. Similarly to Eq. (B1) we can write

$\Pi_{F F \quad(4 \beta, \mu \nu}^{(4 a)}(k)=C_{\mathrm{YM}} \delta^{a b}\left[C_{1}^{(4 a)} I_{\alpha \beta, \mu \nu}+C_{2}^{(4 a)} \frac{1}{k^{2}} L_{\alpha \beta, \mu \nu}(k)\right]$.

Proceeding similarly as in the case of Eq. (B2), we contract the Eq. (B15) with $I_{\mu \nu, \alpha \beta}$ and $L_{\mu \nu, \alpha \beta}(k)$ and solve the system of equations for $C_{1}^{(4 c)}$ and $C_{2}^{(4 c)}$ (as in the previous calculations, this is a very straightforward and well defined computer algebra procedure), yielding the following results

$$
\left\{\begin{array}{lcc}
C_{1}^{(4 a)}= & \frac{\xi+1}{4} g^{2} I^{11} \\
C_{2}^{(4 a)}= & -\frac{1}{16}(d-4)\left(\xi^{2}-1\right) g^{2} I^{11}
\end{array}\right.
$$

Using (B7) (notice that $C_{2}^{(4 a)}$ does not have a pole $1 / \epsilon$ ), we obtain the following result for the UV pole

$$
\Pi_{F F \alpha \beta, \mu \nu}^{(4 a) a b}(k)=i \frac{C_{\mathrm{YM}} g^{2}}{16 \pi^{2} \epsilon} \frac{\xi+1}{4} \delta^{a b} I_{\alpha \beta, \mu \nu}+\cdots
$$

\section{d. $F A$ and $A F$ self-energies}

Finally let us consider the mixed self-energies in Figs. 4(b) and 4(c). In this case, there is just one tensor with three indices and the required symmetry. For instance, in the case of the diagram in Fig. 4(b), the result can be expressed as follows

$$
\Pi_{F A \alpha \beta, \mu}^{(4 b) a b}(k)=C^{(4 b)} C_{\mathrm{YM}} \delta^{a b}\left(k_{\alpha} \eta_{\beta \mu}-k_{\beta} \eta_{\alpha \mu}\right) .
$$

Contracting both sides with the Lorentz structure on the right hand side and performing the integrals with the help of (B6), we obtain

$$
C^{(4 b)}=\frac{i}{8}[d(1-\xi)+3 \xi-1] g^{2} I^{11} .
$$

Using (B7) we obtain the following result for the UV pole

$\Pi_{F A \alpha \beta, \mu}^{(4 b) a b}(k)=\frac{C_{\mathrm{YM}} g^{2}}{16 \pi^{2} \epsilon} \delta^{a b} \frac{\xi-3}{8}\left(k_{\alpha} \eta_{\beta \mu}-k_{\beta} \eta_{\alpha \mu}\right)$.

Proceeding similarly, we obtain

$$
\Pi_{A F \mu, \alpha \beta}^{(4 c) a b}(k)=-\Pi_{F A \alpha \beta, \mu}^{(4 b) a b}(k) .
$$

\section{Propagators}

\section{a. The $F F$ propagator}

Using the results for the self-energies, the propagator for the $F_{\mu \nu}^{a}$ field can be expressed as follows 


$$
\begin{aligned}
D_{F F \mu \nu, \alpha \beta}^{(1) a b}= & D_{F A \mu \nu, \rho}^{(0) a c} \Pi_{A A}^{(1 s t) c d \rho \lambda} D_{A F \lambda, \alpha \beta}^{(0) d b}+D_{F F \mu \nu, \rho \sigma}^{(0) a c} \Pi_{F F}^{(4 a) c d \rho \sigma, \lambda \delta} D_{F F \lambda \delta, \alpha \beta}^{(0) d b} \\
& +D_{F F \mu \nu, \rho \sigma}^{(0) a c} \Pi_{F A}^{(4 b) c d \rho \sigma, \gamma} D_{A F \gamma, \alpha \beta}^{(0) d b}+D_{F A \mu \nu, \rho}^{(0) a c} \Pi_{A F}^{(4 c) c d \rho, \gamma \delta} D_{F F \gamma \delta, \alpha \beta}^{(0) d b} .
\end{aligned}
$$

From the properties of the tensors $L_{\mu \nu, \alpha \beta},\left(k_{\mu} \eta_{\nu \alpha}-k_{\nu} \eta_{\mu \alpha}\right)$ as well as the identity $I_{\mu \nu, \alpha \beta}$ one can show that the last two terms in Eq. (B22) vanish [see Eqs. (A4), (A5) and (A6)]. Using the results for $\Pi_{A A}^{(1 s t) c d \alpha \beta}$ and $\Pi_{F F}^{(4 a) c d \alpha \beta, \gamma \delta}$ given respectively in Eqs. (B13) and (B15) a straightforward calculation yields

$$
D_{F F \mu \nu, \alpha \beta}^{(1) a b}=g^{2} I^{11} C_{\mathrm{YM}} \delta^{a b}\left\{-(\xi+1) I_{\mu \nu, \alpha \beta}+\frac{d[d(\xi-1)-\xi+7]-8}{2(d-1) k^{2}} L_{\mu \nu, \alpha \beta}(k)\right\} .
$$

Using (B7) we obtain the following UV pole part

$$
D_{F F \mu \nu, \alpha \beta}^{(1) a b}=i \frac{C_{\mathrm{YM}} g^{2}}{16 \pi^{2} \epsilon} \delta^{a b}\left\{-(\xi+1) I_{\mu \nu, \alpha \beta}+\left(\frac{2}{3}+2 \xi\right) \frac{1}{k^{2}} L_{\mu \nu, \alpha \beta}(k)\right\}+\cdots
$$

\section{b. The $A A$ propagator}

The one-loop correction to the $A_{\mu}^{a}$ propagator can be calculated from

$D_{A A \mu \nu}^{(1) a b}=D_{A A \mu \alpha}^{(0) a c} \Pi_{A A}^{(1 s t) c d \alpha \beta} D_{A A \beta \nu}^{(0) d b}+D_{A F \mu, \alpha \beta}^{(0) a c} \Pi_{F F}^{(4 a) c d \alpha \beta, \gamma \delta} D_{F A \gamma \delta, \nu}^{(0) d b}+D_{A F \mu, \alpha \beta}^{(0) a c} \Pi_{F A}^{(4 b) c d \alpha \beta, \gamma} D_{A A \gamma \nu}^{(0) d b}+D_{A A \mu \alpha}^{(0) a c} \Pi_{A F}^{(4 c) c d \alpha, \gamma \delta} D_{F A \gamma \delta, \nu}^{(0) d b}$.

Using the results for $\Pi_{A A}^{(1 s t) c d \alpha \beta}, \Pi_{F F}^{(4 a) c d \alpha \beta, \gamma \delta}, \Pi_{F A}^{(4 b) c d \alpha \beta, \gamma}$ and $\Pi_{A F}^{(4 c) c d \alpha, \gamma \delta}$ given respectively in Eqs. (B13), (B15), (B18) and (B21), a straightforward calculation yields

$$
D_{A A \mu \nu}^{(1) a b}=-\frac{d^{2}(1-\xi)(\xi+7)+d(\xi(5 \xi+26)-19)-4 \xi(\xi+5)+16}{8(d-1)} g^{2} I^{11} C_{\mathrm{YM}} \delta^{a b}\left(\frac{\eta_{\mu \nu}}{k^{2}}-\frac{k_{\mu} k_{\nu}}{k^{4}}\right) .
$$

It is immediately clear that Eq. (B26) is the same as the propagator for the $A_{\mu}^{a}$ field in the second-order formalism which can be obtained by simply computing $D_{A A \mu \alpha}^{(0) a c} \Pi_{A A}^{(2 n d) c d \alpha \beta} D_{A A \beta \mu}^{(0) d b}$, where $\Pi_{A A}^{(2 n d) c d \alpha \beta}$ is given by Eq. (B10) [using the transversality, it is easy to see that this will just produce a factor $\left(-1 / k^{4}\right)$ times the self-energy in Eq. (B10)]. This is an explicit special example of the general result, pointed out in Sec. II, according to which the two formalisms give the same Green's functions containing only external $A_{\mu}^{a}$ fields, for any choice of the gauge parameter and dimension $d$. Using (B7) in Eq. (B11), we obtain the following UV pole part

$D_{\text {AA } \mu \nu}^{(1) a b}=i \frac{C_{\mathrm{YM}} g^{2}}{16 \pi^{2} \epsilon} \frac{3 \xi-13}{6} \delta^{a b}\left(\frac{\eta_{\mu \nu}}{k^{2}}-\frac{k_{\mu} k_{\nu}}{k^{4}}\right)+\cdots$.
Using the result for the $A_{\mu}^{a}$ propagator in Eq. (B26), we can now compute the quantity

$$
\left\langle 0\left|T\left(\partial_{\mu} A_{\nu}^{a}-\partial_{\nu} A_{\mu}^{a}\right)(x)\left(\partial_{\alpha} A_{\beta}^{b}-\partial_{\beta} A_{\alpha}^{b}\right)(y)\right| 0\right\rangle,
$$

which is part of the contribution to the right-hand side of Eq. (4.1). The corresponding expression in momentum space $\left(\partial_{\mu} \rightarrow i k_{\mu}\right.$ for the first momentum and $\partial_{\mu} \rightarrow-i k_{\mu}$ for the second momentum) is given by

$k_{\mu} k_{\alpha} D_{A A \nu \beta}^{(1) a b}-k_{\mu} k_{\beta} D_{A A \nu \alpha}^{(1) a b}-k_{\nu} k_{\alpha} D_{A A \mu \beta}^{(1) a b}+k_{\nu} k_{\beta} D_{A A \mu \alpha}^{(1) a b}$,

where we are using that the $A_{\mu}^{a}$ propagator is the same in both formalisms. Using Eq. (B26) we obtain

$$
-\frac{d^{2}(1-\xi)(\xi+7)+d(\xi(5 \xi+26)-19)-4 \xi(\xi+5)+16}{8(d-1)} g^{2} I^{11} C_{\mathrm{YM}} \delta^{a b} \frac{1}{k^{2}}\left(k_{\mu} k_{\alpha} \eta_{\nu \beta}+k_{\nu} k_{\beta} \eta_{\mu \alpha}-k_{\nu} k_{\alpha} \eta_{\mu \beta}-k_{\mu} k_{\beta} \eta_{\nu \alpha}\right)
$$


(notice that terms like $k_{\alpha} k_{\beta} k_{\mu} k_{\nu}$ vanish due to the antisymmetry). Using Eq. (A3), this can be written as

$$
\frac{d^{2}(\xi-1)(\xi+7)-d(\xi(5 \xi+26)+19)+4 \xi(\xi+5)-16}{4(d-1)} g^{2} I^{11} C_{\mathrm{YM}} \delta^{a b} \frac{1}{k^{2}} L_{\mu \nu, \alpha \beta}(k),
$$

which has the following UV pole

$$
i \frac{C_{\mathrm{YM}} g^{2}}{16 \pi^{2} \epsilon} \delta^{a b} \frac{3 \xi-13}{3} \frac{1}{k^{2}} L_{\mu \nu, \alpha \beta}(k) .
$$

The $A_{\mu}^{a}$ propagator in Eq. (B26) can also be used to compute the quantity

$$
\left\langle 0\left|T\left(\partial_{\mu} A_{\nu}^{a}-\partial_{\nu} A_{\mu}^{a}\right)(x) A_{\alpha}^{b}(y)\right| 0\right\rangle,
$$

that appears on the right-hand side to the identity in Eq. (3.1). In momentum space, $\left(\partial_{\mu} \rightarrow i k_{\mu}\right)$ this becomes

$$
i k_{\mu} D_{A A \nu \alpha}^{(1) a b}-i k_{\nu} D_{A A \mu \alpha}^{(1) a b}
$$

Using Eq. (B26), we obtain

$$
\begin{aligned}
& i \frac{d^{2}(\xi-1)(\xi+7)-d(\xi(5 \xi+26)-19)+4 \xi(\xi+5)-16}{8(d-1)} \\
& \quad \times g^{2} I^{11} C_{\mathrm{YM}} \delta^{a b} \frac{1}{k^{2}}\left(k_{\mu} \eta_{\nu \alpha}-k_{\nu} \eta_{\mu \alpha}\right),
\end{aligned}
$$

which has the following UV pole part

$$
\frac{C_{\mathrm{YM}} g^{2}}{16 \pi^{2} \epsilon} \frac{13-3 \xi}{6} \frac{1}{k^{2}}\left(k_{\mu} \eta_{\nu \alpha}-k_{\nu} \eta_{\mu \alpha}\right)+\cdots
$$

\section{c. The $F A$ and $A F$ propagators} by

The one-loop contribution to the $F A$ propagator is given

$$
\begin{aligned}
D_{F A \mu \nu, \alpha}^{(1) a b}= & D_{F F \mu \nu, \rho \sigma}^{(0) a c} \Pi_{F F}^{(4 a) c d \rho \sigma, \lambda \delta} D_{F A \lambda \delta, \alpha}^{(0) d b} \\
& +D_{F F \mu \nu, \rho \sigma}^{(0) a c} \Pi_{F A}^{(4 b) c d \rho \sigma, \gamma} D_{A A \gamma, \alpha}^{(0) d b} \\
& +D_{F A \mu \nu, \rho}^{(0) a c} \Pi_{A A}^{(1 s t) c d \rho \lambda} D_{A A \lambda, \alpha}^{(0) d b} \\
& +D_{F A \mu \nu, \rho}^{(0) a c} \Pi_{A F}^{(4 c) c d \rho, \gamma \delta} D_{F A \gamma \delta, \alpha}^{(0) d b}
\end{aligned}
$$

Using the results form the self-energies and the free propagators, the first two terms in Eq. (B37) vanish [see Eqs. (A4), (A5) and (A6)] and the sum of the last two terms yields

$$
\begin{aligned}
D_{F A \mu \nu, \alpha}^{(1) a b}= & i \frac{[d(2 d-7)(\xi-1)+5 \xi-7]}{4(d-1)} \\
& \times g^{2} I^{11} C_{\mathrm{YM}} \delta^{a b} \frac{1}{k^{2}}\left(k_{\mu} \eta_{\nu \alpha}-k_{\nu} \eta_{\mu \alpha}\right) .
\end{aligned}
$$

Using (B7) we obtain the following UV divergent result $D_{F A \mu \nu, \alpha}^{(1) a b}=\frac{C_{\mathrm{YM}} g^{2}}{16 \pi^{2} \epsilon} \delta^{a b} \frac{1}{k^{2}} \frac{11-9 \xi}{12}\left(k_{\mu} \eta_{\nu \alpha}-k_{\nu} \eta_{\mu \alpha}\right)+\cdots$.

Proceeding similarly, we obtain the following result for the $A F$ propagator

$$
D_{A F \alpha, \mu \nu}^{(1) a b}=-D_{F A \mu \nu, \alpha}^{(1) a b}
$$

\section{Pinched diagrams}

Let us first consider the diagram in Fig. (1b). This arises from the nonlinear part $\left\langle 0\left|T f^{a c d} A_{\mu}^{c}(x) A_{\nu}^{d}(x) A_{\alpha}^{b}(y)\right| 0\right\rangle$ of $\left\langle 0\left|T f_{\mu \nu}^{a}(x) A_{\alpha}^{b}(y)\right| 0\right\rangle$ [the linear part has been taken into account in Eq. (B33), which has the momentum space result given in Eq. (B35)]. Since this contribution involves the product of two fields at the same space-time point $x$, in the momentum space they become loop like diagrams, containing one cubic vertex, given by (A8a), and two propagators given by (A1b). As we can see in Fig. 6, the momentum structure is similar to the one shown in the graph of Fig 4(b) but with no vertex on the left and replacing the $A F$ internal line by a $A A$ line. Also, there is a free propagator on the right side. Therefore, the corresponding expression in momentum space have the same tensor structure as in Eq. (A1d). Proceeding as in Sec. $1 \mathrm{~d}$, we find

$$
\begin{gathered}
\frac{i}{8}[d(1-\xi)(\xi+3)+2 \xi(2 \xi+5)-2] \\
\quad \times g^{2} I^{11} C_{\mathrm{YM}} \delta^{a b} \frac{1}{k^{2}}\left(k_{\mu} \eta_{\nu \alpha}-k_{\nu} \eta_{\mu \alpha}\right)
\end{gathered}
$$

which has the following UV pole part

$$
-\frac{C_{\mathrm{YM}} g^{2}}{16 \pi^{2} \epsilon} \delta^{a b} \frac{\xi+5}{4} \frac{1}{k^{2}}\left(k_{\mu} \eta_{\nu \alpha}-k_{\nu} \eta_{\mu \alpha}\right)+\ldots
$$

Adding the results in Eqs. (B41) and (B35), we obtain (B38), which confirms the identity (3.1) to one loop order. 


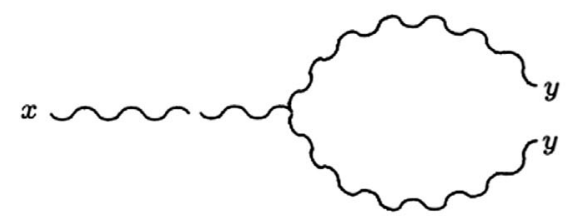

(a)

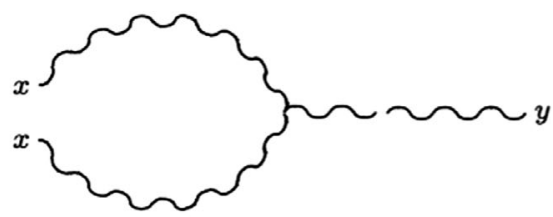

(b)

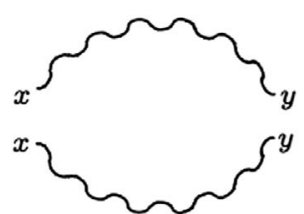

(c)
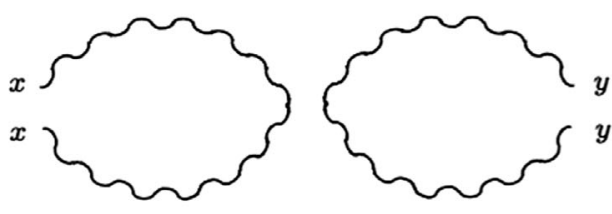

(d)

FIG. 5. "Pinched" contributions from $\left\langle 0\left|T\left(\partial_{\mu} A_{\nu}^{a}(x)-\partial_{\nu} A_{\mu}^{a}(x)\right) f^{b c d} A_{\alpha}^{c}(y) A_{\beta}^{d}(y)\right| 0\right\rangle,\left\langle 0\left|T f^{a c d} A_{\mu}^{c}(x) A_{\nu}^{d}(x)\left(\partial_{\alpha} A_{\beta}^{b}(y)-\partial_{\beta} A_{\alpha}^{b}(y)\right)\right| 0\right\rangle(\mathrm{a}$ and b) and $\left\langle 0\left|T f^{a c d} A_{\mu}^{c}(x) A_{\nu}^{d}(x) f^{b e g} A_{\alpha}^{e}(y) A_{\beta}^{g}(y)\right| 0\right\rangle$ (c). There is a second diagram identical to (c) which can be obtained by interchanging the $x$ points. The graphs in (d) vanish upon using dimensional regularization in momentum space.

Figure 5(b) shows the contributions from $\langle 0| T f^{a c d} A_{\mu}^{c}(x) \times$ $A_{\nu}^{d}(x)\left(\partial_{\alpha} A_{\beta}^{b}(y)-\partial_{\beta} A_{\alpha}^{b}(y)\right)|0\rangle$ which arises from the nonlinear parts of $\left\langle 0\left|T f_{\mu \nu}^{a}(x) f_{\alpha \beta}^{b}(y)\right| 0\right\rangle$ [the linear part was considered in Eq. (B28), which has the momentum space result given in Eq. (B31)]. Similarly to the previous calculation of the graphs in Fig. 1(b), the products of fields at the same space-time point $x$ give rise to loop integrals. As shown in Fig. 6(a), the momentum space expression is a one-loop diagram similar to the contribution from 1(b), but in this case the basic graph is contracted with $g f^{a c d}\left[\left(-i k_{\alpha}\right) D_{A A \gamma \beta}^{(0) e b}(k)-\alpha \leftrightarrow \beta\right]$. Since the corresponding expressions in momentum space have the same tensor structure as the $F F$-propagator, we can proceed as in Sec. B $1 \mathrm{c}$. Using the same tensor basis formed with the tensors in Eqs. (A2) and (A3) we obtain for the sum of the contributions from Figs. 1(a) and 1(b) the following result

$$
\begin{aligned}
& g^{2} I^{11} C_{\mathrm{YM}} \delta^{a b}\left[\frac{1}{2} d(1-\xi)(\xi+3)+\xi(2 \xi+5)-1\right] \\
& \quad \times \frac{1}{k^{2}} L_{\mu \nu, \alpha \beta}(k),
\end{aligned}
$$

which has the following UV pole

$$
i \frac{g^{2} C_{\mathrm{YM}}}{16 \pi^{2} \epsilon} \delta^{a b}(\xi+5) L_{\mu \nu, \alpha \beta}(k)+\cdots .
$$

Finally, we have the contribution from $\langle 0| T f^{a c d} A_{\mu}^{c}(x) \times$ $A_{\nu}^{d}(x) f^{b e g} A_{\alpha}^{e}(y) A_{\beta}^{g}(y)|0\rangle$ in $\left\langle 0\left|T f_{\mu \nu}^{a}(x) f_{\alpha \beta}^{b}(y)\right| 0\right\rangle$ which is shown in Fig. 5(c) (there is also an identical contribution obtained by interchanging the two $x$ points). Similarly to the previous cases, there is a momentum space expression with a single loop associated with this contribution. As before, the loop is associated with the pinch of the

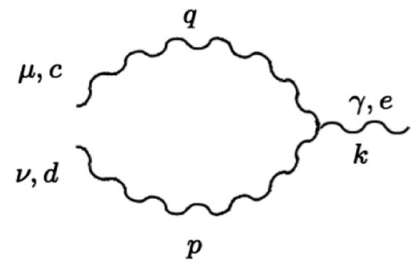

(a)

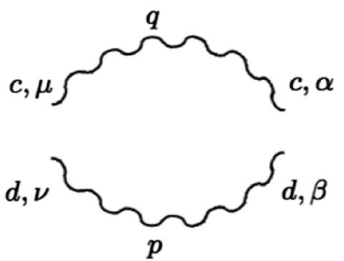

(b)
FIG. 6. The two basic momentum space graphs associated with Figs. 1(b) and (5). The momentum space expressions associated with 1(b) and 5(b) are obtained upon contracting (a) with $g f^{a c d} D_{A A \gamma \alpha}^{(0) e b}(-k)$ and $g f^{a c d}\left[\left(-i k_{\alpha}\right) D_{A A \gamma \beta}^{(0) e b}(-k)-\alpha \leftrightarrow \beta\right]$ respectively. The momentum space expression associated with $5(\mathrm{c})$ is obtained upon contracting (b) with $g^{2} f^{a c d} f^{b c d}$.

propagators at the same space-time point. However, in this case we have pinches at both sides so that there is no interaction vertex. The corresponding loop diagram is shown in Fig. 6(b). In terms of the tensors $I_{\mu \nu, \alpha \beta}$ and $L_{\mu \nu, \alpha \beta}(k)$, we obtain the following result for the loop integral

$$
\begin{aligned}
& g^{2} I^{11} C_{\mathrm{YM}} \delta^{a b}\left[-(\xi+1) I_{\mu \nu, \alpha \beta}+\frac{1}{4}(d-4)\left(\xi^{2}-1\right)\right. \\
& \left.\quad \times \frac{1}{k^{2}} L_{\mu \nu, \alpha \beta}(k)\right]
\end{aligned}
$$

which has the following UV pole

$$
-i \frac{g^{2} C_{\mathrm{YM}}}{16 \pi^{2}} \delta^{a b}(\xi+1) I_{\mu \nu, \alpha \beta} .
$$

Adding the Eqs. (B31), (B43) and (B45), a straightforward algebra shows that the result is the same as (B23), so that the identity (4.1) is verified to one-loop order (of course this remains true for the UV pole part). 
[1] S. Okubo and Y. Tosa, Phys. Rev. D 20, 462 (1979); 23, 1468(E) (1981).

[2] D. G. C. McKeon, Can. J. Phys. 72, 601 (1994).

[3] M. Martellini and M. Zeni, Phys. Lett. B 401, 62 (1997).

[4] K. Costello, Renormalisation and Effective Field Theory, Mathematical Surveys and Monographs (American Mathematical Society, Providence, Rhode Island, 2011).

[5] F. T. Brandt and D. G. C. McKeon, Phys. Rev. D 91, 105006 (2015).

[6] F. T. Brandt and D. G. C. McKeon, Phys. Rev. D 93, 105037 (2016).

[7] J. Frenkel and J. C. Taylor, Ann. Phys. (Amsterdam) 387, 1 (2017).

[8] J. Frenkel and J. C. Taylor, Ann. Phys. (Amsterdam) 389, 234 (2018).
[9] F. T. Brandt, J. Frenkel, and D. G. C. McKeon, Ann. Phys. (Amsterdam) 409, 167932 (2019).

[10] I. L. Buchbinder and P. M. Lavrov, Eur. Phys. J. C 78, 524 (2018).

[11] P. M. Lavrov, arXiv:2002.05997.

[12] A. O. Barvinsky, D. Blas, M. Herrero-Valea, S. M. Sibiryakov, and C. F. Steinwachs, J. High Energy Phys. 07 (2018) 035.

[13] K. G. Wilson and W. Zimmermann, Commun. Math. Phys. 24, 87 (1972).

[14] T. Muta, Foundations of Quantum Chromodynamics (World Scientific, Singapore, 1987).

[15] S. Weinberg, Quantum Theory of Fields II (Benjamin Cummings, Cambridge, 1995). 